\title{
We Perceive Music before we Understand Speech
}

\author{
Hendrik Purwins \\ Music Technology Group, Universitat Pompeu Fabra, Barcelona, Spain \\ and \\ Sound Perception and Design Group, Institut de Recherche et Coordination \\ Acoustique/Musique, Paris, France
}

Keywords: music and emotion, evolution of music, music cognition

In his article "Musical emotions: Functions, origins, evolution" [1], Perlovsky explains "concept-models" with the analogy of the projection of memories and representations onto the visual cortex. He postulates the principle of "knowledge instinct" that aims at matching concept-models to the surroundings. According to Perlovsky, this instinct causes emotions, satisfaction or dissatisfaction, depending on whether the world and our knowledge about the world coincide. In a hierarchy of concepts, each higher level gives a meaning to the level below. He claims that emotions and concepts have separated for humans. Languages balance between both aspects. By "differentiation", he denotes the generation of more specific, detailed concepts, whereas "synthesis" is performed by the knowledge instinct, creating meanings and purpose on higher levels. For a "knowledge-accumulating" dynamic culture, or as a special case, music evolution, Perlovsky suggests a dialectic theory, an oscillation between high and low differentiation and synthesis. In the tradition of an integral humanistic view of cultural, aesthetic and musical evolution, Perlovsky's theory claims wide generalizability. Its formulation as a testable hypothesis poses a challenge.

Also in the evolution of the individual human being, there is a state of unity between concept and emotion. Among the first concepts babies can distinguish are the emotional states of their mothers. If we consider rhythm and pitch contours to be among the main constituting features of music, we could say that a baby learns language through music. If their mother is loving/comfortable, fearful or surprised is reflected in the rhythmical pitch contour of the mother's speech [2,3]. Mean pitch, mean pitch range, mean syllable duration characterize the different emotions. The infant learns to 
distinguish emotions before any other speech feature. As soon as a word is recognized, our perception is determined by the meaning of the utterance or sound more than by its acoustical and musical attributes.

[1] L. Perlovsky, Musical emotions, cognitive science, and art of music, Physics of Life Reviews 7 (1) (2010) 49 - 54.

[2] H. Purwins, P. Herrera, M. Grachten, A. Hazan, R. Marxer, X. Serra, Computational models of music perception and cognition I: The perceptual and cognitive processing chain, Physics of Life Reviews 5 (3) (2008) $151-168$.

[3] L. J. Trainor, C. M. Austin, R. N. Desjardins, Is infant-directed speech prosody a result of vocal expression of emotion?, Psychological Science 11 (3) (2000) 188-195. 